\title{
Le mètre et le tāl dans la musique de l'Inde du Nord
}

Metre and tāl in North Indian music

\section{Martin Clayton}

Traducteur : Georges Goormaghtigh

\section{OpenEdition \\ Journals}

Édition électronique

URL : http://journals.openedition.org/ethnomusicologie/888

ISSN : 2235-7688

Éditeur

ADEM - Ateliers d'ethnomusicologie

Édition imprimée

Date de publication : 1 décembre 1997

Pagination : 169-189

ISBN : 2-8257-0579-9

ISSN : 1662-372X

Référence électronique

Martin Clayton, «Le mètre et le tāl dans la musique de l'Inde du Nord », Cahiers d'ethnomusicologie [En ligne], 10 | 1997, mis en ligne le 06 janvier 2012, consulté le 19 avril 2019. URL : http://

journals.openedition.org/ethnomusicologie/888

Ce document a été généré automatiquement le 19 avril 2019

Tous droits réservés 


\title{
Le mètre et le tāl dans la musique de l'Inde du Nord
}

\author{
Metre and tāl in North Indian music
}

Martin Clayton

Traduction : Georges Goormaghtigh

\section{NOTE DE L'ÉDITEUR}

Traduit de l'anglais par Georges Goormaghtigh.

1 La musique savante de l'Inde du Nord (hindoustānī) possède une théorie métrique sophistiquée, appelée tâl, dont une série de traités musicologiques nous permettent de retracer le développement continu sur une période d'à peu près deux millénaires. Cette théorie décrit l'organisation temporelle de toute la musique mesurée (nibaddh) appartenant à la tradition classique de l'Inde du Nord; la musique kharnatique de l'Inde du Sud possède son propre système de tāl qui s'est développé de façon autonome depuis trois à quatre cents ans. La relation entre la théorie et la pratique de cette musique est évidemment complexe et fut l'objet d'une étude antérieure ${ }^{2}$. Nous reprenons ici certaines des conclusions de cette étude - qui décrit la pratique du tāl en rapport avec sa théorie plutôt que de s'intéresser à la seule théorie - afin de discuter du tãl dans le contexte des autres musiques et des théories ethnomusicologiques du rythme et du mètre.

2 Nous nous proposons tout d'abord de chercher à savoir si des théories musicologiques ou psychologiques, à l'origine sans rapport avec l'étude de la musique indienne, sont susceptibles d'apporter des lumières sur le tāl, et ensuite de voir en quoi l'étude du tâl peut contribuer au développement des théories générales du rythme et du mètre en ethnomusicologie ${ }^{3}$. Nous verrons que certains aspects du tāl peuvent être décrits en termes de théories générales puisqu'ils correspondent à des éléments similaires dans différents systèmes métriques. D'autres aspects sont plus rares et probablement spécifiques à la musique du Nord de l'Inde, ce qui ne devrait pas nous surprendre dans la 
mesure où chaque système métrique peut, dans un certain sens, représenter un cas particulier. Des questions plus générales se profilent derrière une telle recherche : en rassemblant un nombre suffisant de données et d'analyses de cultures musicales différentes, serait-il possible de développer des théories du rythme et du mètre applicables de façon générale et à travers lesquelles chaque système particulier, bien qu'envisagé dans sa spécificité, révélerait des données de base explicables en termes généraux ? Chaque système rythmique peut-il être décrit selon des paramètres se fondant sur des universaux psycho-physiologiques, dans des limites localement déterminées pour chaque paramètre ? Ou alors la diversité des rythmes musicaux est-elle trop grande pour en envisager une étude aussi générale ou une approche comparative? Si cette dernière hypothèse est la bonne (et jusqu'à preuve du contraire beaucoup le penseront), peut-on encore définir objectivement des termes tels que syncope, polyrythmie, polymétrie, voire même les notions de tempo, de mètre et de rythme? Comment utiliser des termes et des concepts occidentaux dans les analyses rythmiques et les transcriptions de musique nonoccidentales, sans être égaré par un tel ethnocentrisme?

Si nous voulons approfondir l'étude du rythme musical, il faut commencer par reconstruire la théorie rythmique à partir de ses principes de base, une reconstruction qui, espérons-le, produira des paramètres et des concepts rythmiques objectivement définissables grâce auxquels s'ouvrira un vaste champ d'études. Sans de tels instruments on sera toujours submergé sous des montagnes de données difficilement analysables de façon sûre, et l'analyse rythmique restera aussi peu développée et peu utilisée qu'elle l'a toujours été en ethnomusicologie. Comme nous allons tenter de le montrer, nous croyons que différentes théories et réflexions issues des recherches dans le domaine de la psychologie cognitive peuvent être utiles à cette étude : on pourrait donc dire que cet article s'inscrit dans une perspective d'ethnomusicologie cognitive.

Nous ne traiterons qu'un aspect de ce problème. Tout d'abord nous rappelerons les grandes lignes des théories métriques utilisées par les ethnomusicologues puis, tout en gardant celles-ci présentes à l'esprit, nous aborderons le tāl dans la musique de l'Inde du Nord et nous discuterons des implications du tāl pour l'ethnomusicologie et vice versa.

\section{La métrique : une perspective ethnomusicologique}

5 Le mètre, avec le tempo et le rythme lui-même, est un des concepts-clé utilisés pour décrire le phénomène rythmique. Mais qu'est-ce que le mètre? Pour beaucoup, en particulier pour ceux qui connaissent la notation sur portée, il s'agit simplement de la dimension de la musique représentée par le chiffrage de la mesure. Un tel chiffrage est formé de deux composantes, l'une décrit le nombre de temps par mesure et l'autre le signe utilisé pour noter chacun de ces temps. Le chiffrage de la mesure indique donc qu'une unité de temps est spécifiée comme pulsation ${ }^{4}$ et qu'un nombre défini de ces dernières sont groupées pour former une mesure. Dans presque toute la musique savante occidentale, le premier temps d'une mesure est le plus fort, et il est suivi d'un ou plusieurs temps subsidiaire forts ou «faibles ». Le mètre est généralement décrit par les musicologues comme une succession de pulsations fortes et faibles ou comme un regroupement de pulsations pour mesurer le temps ${ }^{5}$.

Pour le musicien et le musicologue occidental, le mètre ne semble donc être qu'un simple concept, supposition qui se confirme lorsqu'on constate la brièveté des rubriques consacrées à ce mot dans des dictionnaires musicaux tels que le New Grove (Grove 6, 
1980). Notre but n'est pas d'examiner en détail la notion telle qu'elle est utilisée en musique occidentale ${ }^{6}$, bien qu'il faille remarquer que certains musicologues ne sont pas nécessairement d'accord sur la similarité du mètre avec le chiffrage de la mesure ${ }^{7}$.

7 Sans plus s'attarder sur la notion de mètre dans la musique savante occidentale, on peut s'interroger sur le bien-fondé de l'hypothèse de nombreux ethnomusicologues que le mètre serait un simple concept applicable à un vaste éventail de musiques, toutes susceptibles d'être transcrites selon le système de mesure de la musique occidentale. Nous ne contestons par le fait que le chiffrage de la mesure puisse parfois fonctionner dans la description de musiques non-occidentales. Ce que nous contestons, c'est son application sans examen attentif des questions qui s'y rattachent. Les problèmes qu'ont à affronter les ethnomusicologues en notant les rythmes de musiques des quatre coins du monde sont de taille, même si beaucoup d'entre eux n'en ont pas vraiment conscience ${ }^{8}$. L'insuffisance des résultats est d'habitude liée à une incapacité de traiter des problèmes qui surgissent inévitablement lorsqu'on tente d'adapter une musique non-occidentale à des conventions d'écriture occidentales. Dans la musique savante occidentale, bien que la pratique ait pu varier avec les époques et les compositeurs, on ne peut prétendre que le chiffrage de la mesure corresponde toujours à la métrique telle quelle est perçue par l'ensemble des auditeurs ou la plus grande partie d'entre eux. Les ethnomusicologues devraient donc se mettre d'accord pour savoir si le chiffrage de la mesure (un mètre noté) utilisé dans une transcription est là pour indiquer une métrique perçue (et si tel et le cas, par qui) ou si elle sert seulement d'aide à la lecture, sans signification métrique réelle.

8 Laissons de côté pour l'instant la musique apparemment non-mesurée ou de «rythme libre $»^{9}$ pour ne nous intéresser qu'à la musique clairement mesurée. Si l'on veut représenter un tel rythme en notation sur portée, quelle unité de temps correspondra à la "pulsation » et comment la noter? Est-ce que le groupement de deux, trois ou quatre pulsations sont suffisants pour déterminer une mesure, ou alors des regroupements à un plus haut niveau (de six, huit, douze voir même seize pulsations) ont-ils un sens du point de vue du mètre? Où commence et où se termine une mesure et quelle pulsation correspond à un «temps " ou à un " contre-temps »? Toutes ces décisions peuvent être lourdes de conséquences dans les transcriptions ethnomusicologiques. Sans compter que la notation sur portée est fortement influencée par des conceptions métriques originaires d'Occident, le premier temps d'une mesure étant par exemple nécessairement un temps « fort ». Même l'idée, apparemment fondamentale, que les notes doivent être considérées comme des événements se déployant dans la durée (ce qui est implicite dans notre système de notation), et comme des événements intervenant en des points précis du développement temporel n'est peut-être pas partagée de façon universelle.

9 La question du mètre et de sa représentation en ethnomusicologie devrait être clairement posée. Agawu (1995) prend pourtant le contre-pied de cette affirmation en estimant que, souvent, les chercheurs font preuve de trop de réserve dans l'utilisation du chiffrage de la mesure pour la musique africaine, et ont recours à des transcriptions d'une polymétrie inutilement complexe ou à des notations inventées de toute pièce pour éviter ce problème $\mathrm{e}^{10}$. L'argument d'Agawu est certainement convaincant dans la mesure où, là où il y a de bonnes raisons de représenter une interprétation métrique particulière, l'utilisation de la notation sur portée et du chiffrage de la mesure peut être la façon la plus efficace de procéder. Mais même en admettant la chose - et sans se laisser entraîner dans des considérations d'ordre idéologique sur le statut hégémonique de la notation sur portée - les problèmes pratiques restent nombreux car beaucoup de pièces musicales ne 
peuvent aisément être interprétées selon un schéma métrique : Les représenter au moyen de symboles conventionnels de la notation musicale risque de fourvoyer le lecteur. Dans ces cas là, modifier la notation occidentale, voire même s'en détourner, ne devrait pas être simplement interprété comme une sorte d'exotisme post-colonial.

Il y a manifestement un besoin de clarifier le concept du mètre pour disposer de termes définissables et dotés d'un sens précis avec lesquels décrire différentes musiques, ainsi que des techniques de notation applicables au plus grand nombre possible d'entre elles. Trois recherches importantes ont permis de faire des progrès dans ce sens.

11 Tout d'abord, nous devons beaucoup à Kolinski pour les connexions qu'il a établies entre le mètre et la Gestalt Psychologie (1973). Il décrit le mètre comme étant le fond sur lequel la surface rythmique est perçue. Selon ce point de vue, le mètre serait une sorte de grille de référence qui influencerait profondément la perception et la connaissance du rythme, la « figure » rythme par rapport au «fond $»^{11}$. Bien entendu, dans la plupart des cas, le mètre doit lui-même être inféré de la surface rythmique par l'auditeur, ce qui suggère un mécanisme complexe de la connaissance musicale dans lequel le mètre est déduit de façon subjective de la surface rythmique qui, à son tour, est interprétée en fonction justement de ce cadre métrique.

Dans un deuxième temps, Lerdahl et Jackendoff (1983), en tentant de démêler métrique et structures de regroupement dans la musique tonale occidentale, sont parvenus à développer la plus convaincante des théories métriques proposées jusqu'à nos jours en musicologie, bien qu'on ne puisse encore savoir dans quelle mesure elle est applicable à d'autres musiques. Dans cette théorie, le mètre est décrit en termes d'interaction de différents niveaux de pulsation concurrents, au nombre de deux ou plus, pour produire des temps relativement forts ou faibles (la notion de temps « forts » doit s'entendre dans un sens structural abstrait et ne correspond pas nécessairement à des sons plus intenses ou accentués que les temps «faibles»). Un point dans le temps perçu comme battement sur deux niveaux de pulsations différents sera "structurellement plus fort» que celui ressenti comme battement sur un niveau seulement. Pour qu'une musique ait une métrique, il faut donc qu'elle soit perçue comme ayant au moins deux niveaux de pulsation : la plupart du temps il y en a trois ou plus. Cette analyse et la notation par points utilisées pour l'illustrer s'avèrent être de puissants instruments d'analyse en musique tonale occidentale et peuvent peut-être s'adapter à d'autres contextes ${ }^{12}$.

Ainsi, avec l'image du mètre de Kolinski comme arrière-plan cognitif ou cadre du rythme et la théorie de la nature du mètre et du système de notation et d'analyse de Lerdahl et Jackendoff, nous avons l'ébauche d'un concept métrique susceptible d'être appliqué à grande échelle. Tout cela doit cependant être vu à la lumière d'une troisième approche, peut-être plus radicale encore : celle d'Arom (1991). Ce dernier suggère que, bien que la polyrythmie centrafricaine soit clairement organisée de façon périodique, le concept et le terme de "mètre" sont inappropriés dans un tel contexte. Si le mètre implique une hiérarchie de pulsations fortes et faibles la polyrythmie centrafricaine, elle, consiste en un réseau de motifs rythmiques périodiques qui s'entrecroisent et s'organisent autour d'un seul niveau primaire de pulsation. Comme il n'y a qu'un seul niveau de pulsation, et pas de hiérarchie de temps ou de "temps premier» cette organisation ne peut être décrite en termes de mètre.

L'analyse perspicace d'Arom nous fournit un autre outil précieux. Selon Lerdahl, Jackendoff et d'autres ${ }^{13}$, le mètre est une hiérarchie de temps «forts » et "faibles » par rapport auxquels le rythme est interprété. Mais comme le montre Arom, le rythme peut 
être organisé différemment - et notre concept de mètre doit donc être redéfini de façon plus souple, ou alors, comme le propose Arom, nous devons développer de nouveaux concepts et de nouvelles théories pour décrire l'organisation rythmique non métrique. Les travaux d'Arom nous invitent à trouver des concepts plus fondamentaux de l'organisation rythmique, mais ne nous obligent pas à abandonner le concept de métrique. En fait, nous pouvons encore affiner ce concept, sans toutefois nous enfermer dans l'hypothèse déraisonnable qu'il est applicable à toutes les musiques.

De nombreuses cultures possèdent des formes musicales qui ne semblent pas être organisées de façon périodique (ce qu'on appelle le « rythme libre »). En outre, comme l'a montré Arom, même des musiques organisées de façon périodique peuvent échapper à une description en terme de mètre. Le mètre n'est manifestement pas un concept universel. On peut néanmoins en envisager l'utilisation du concept de mètre hors de sa culture d'origine puisque l'organisation du rythme en fonction d'un motif périodique de temps « forts » et « faibles » n'est certainement pas limitée à la musique occidentale. Pour ce faire on doit tenir compte de la complexité de la métrique dans la performance et la connaissance musicales. Le premier pas dans cette direction est d'admettre que le mètre est avant tout une construction mentale, un aspect de la représentation cognitive de la musique.

Les recherches en psychologie cognitive de la musique ont déjà commencé à mettre en évidence la complexité de la relation entre les ondes sonores et le mètre, entre le son musical et sa représentation cognitive. La construction d'une représentation cognitive du mètre serait donc loin d'être un processus simple se fondant sur l'identification des sons les plus puissants. Tant que nous n'en saurons pas plus sur la façon dont cette reconstruction prend place et varie, selon le contexte musical et culturel de l'auditeur ${ }^{14}$, nous devons admettre que notre compréhension du mètre en tant que phénomène général est incomplète. Beaucoup reste à explorer dans de futures recherches, aussi bien en psychologie cognitive qu'en ethnomusicologie. Il nous faut cependant évaluer nos connaissances actuelles et proposer des hypothèses susceptibles d'être affinées dans le futur. Les propositions suivantes constituent, selon nous et pour l'instant, une donnée raisonnable sur le mètre qui pourra servir de point de référence pour la musique de l'Inde du Nord.

1. Beaucoup de musiques (mais pas toutes) sont organisées en fonction d'un cadre temporel périodique et hiérarchique, si bien qu'une représentation cognitive de ce cadre peut se former dans l'esprit de l'auditeur. Cette organisation et sa représentation sont appelées « la métrique $"$.

2. On peut dire qu'il y a mètre lorsqu'on perçoit l'interaction d'au moins deux courants continus de pulsations; ces courants sont composés de points dans le temps (battements) séparés par des unités de temps catégoriquement équivalentes. Les points dans le temps perçus comme des battements sur plus d'un niveau sont plus «forts » que ceux qui ne sont des battements que pour un seul niveau. La série de battements forts et faibles qui en résulte est à la fois hiérarchique et périodique.

3. La relation entre mètre et rythme a deux aspects principaux et complémentaires: le mètre est perçu (en partie ou tout à fait subjectivement) sur la base de l'évidence que présente le rythme ${ }^{15}$ alors que le rythme est interprété selon sa relation à ce mètre ${ }^{16}$.

4. La perception d'un mètre est un phénomène complexe qui est influencé par les expériences et la formation musicale de l'auditeur, et plus indirectement peut-être, par son expérience générale et son milieu culturel. Par conséquent la théorie métrique et la pratique sont dans 
une grande mesure déterminées culturellement bien qu'elles reposent en définitive sur les mêmes universaux psycho - physiologiques.

\section{Le tāl : le mètre dans la musique de l'Inde du Nord}

Le tāl est un sujet bien trop complexe pour être décrit ici en entier. Notre intention est d'exposer brièvement une théorie générale du tāl accompagnée de certaines remarques sur la relation entre cette théorie et la pratique de la musique de l'Inde du Nord. Nous résumerons cette relation en disant simplement que la théorie a joué un rôle important dans le développement de cette pratique, et qu'en fait, tel qu'il est couramment pratiqué, le tāl n'aurait pas pu se développer sans le soutien de la théorie. Il est également vrai cependant que la théorie ne rend pas toujours entièrement ou adéquatement compte de la pratique. Le mètre musical ne peut être compris de façon satisfaisante qu'à travers la théorie du tāl, et pourtant cette théorie peut avoir l'effet d'un verre déformant. En particulier, elle rend la tradition apparemment plus homogène et plus liée à des précédents historiques qu'elle ne l'est en réalité.

Selon la théorie musicale indienne, la musique peut être soit anibaddh (non liée, c'est-àdire sans tāl) soit nibaddh (liée, avec tāl). Les formes nibaddh doivent être organisées selon une des structures métriques autorisées ou tāl. Chaque tāl (la musique savante de l'Inde du Nord en comporte à peu près une vingtaine qui sont couramment utilisés) est fondé sur l'interaction de trois niveaux de pulsations appelés mātrā (battements), āvart (cycle) et vibhāg (section). Un certain nombre de mātrā, d'habitude entre six et seize constitue un $\bar{a}$ vart ou cycle, et ce cycle se divise en plusieurs vibhāg ou subdivisions qui ne sont pas nécessairement d'égale longueur. Autrement dit, plusieurs vibhāg comportant chacun plusieurs mātrā s'additionnent pour former un āvārt.

Dans le jhaptāl par exemple, chaque āvārt comporte dix mātrā, répartis en quatre vibhāg de respectivement 2, 3, 2 et 3 mātrā. Cette structure peut être illustrée ainsi :

Fig. 1 : Description de jhaptāl selon trois importants niveaux de pulsation.

Jhaptāl: 10 mātrā, $2+3+2+3$

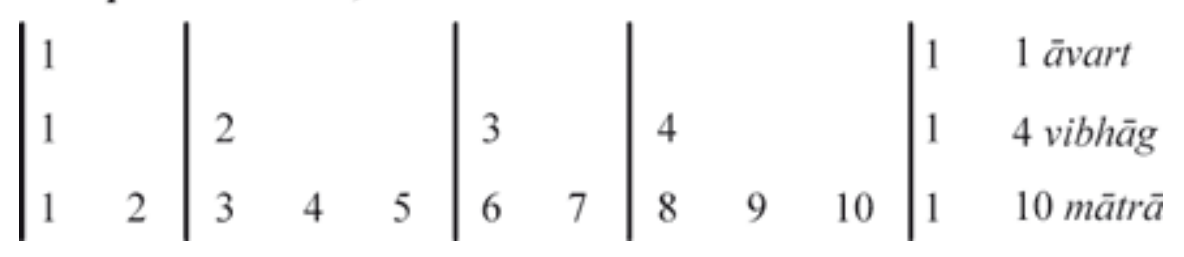

20 La structure du tāl peut être indiquée lors de l'exécution par le musicien et /ou par les auditeurs, en marquant le premier mātrā de chaque vibhāg avec des gestes de la main. Deux gestes principaux sont utilisés à cet effet : tālì (battue) indiqué dans la notation par +, et khālī (geste muet) noté par 0. Le premier temps, sam, malgré son importance structurelle en musique, ne nécessite pas de geste spécifique ${ }^{17}$ puisqu'il est déterminé sans ambiguïté par la nature asymétrique du système de battue: les musiciens et les auditeurs ayant une formation musicale savent quel geste de la séquence représente le sam. 
Fig. 2 : jhaptāl, schéma de la séquence des gestes exprimant le rythme

Jhaptāl: 10 mätrā, $2+3+2+3$

\begin{tabular}{|ll|lll|ll|lll|l}
+ & & + & & & 0 & & + & & & + \\
modèle de battue
\end{tabular}

(+ =battue, $\mathrm{O}=$ geste muet $)$.

21 En plus de ce schéma chironomique, chaque tāl possède une séquence de frappes sur le tambour appelée thekā, qui définit partiellement le tāl et est souvent le moyen le plus sûr pour l'identifier dans la pratique.

Fig. 3 : jhaptāl, schéma indiquant les battues et le țhekā.

Jhaptāl: 10 mātrā, 2+3+2+3

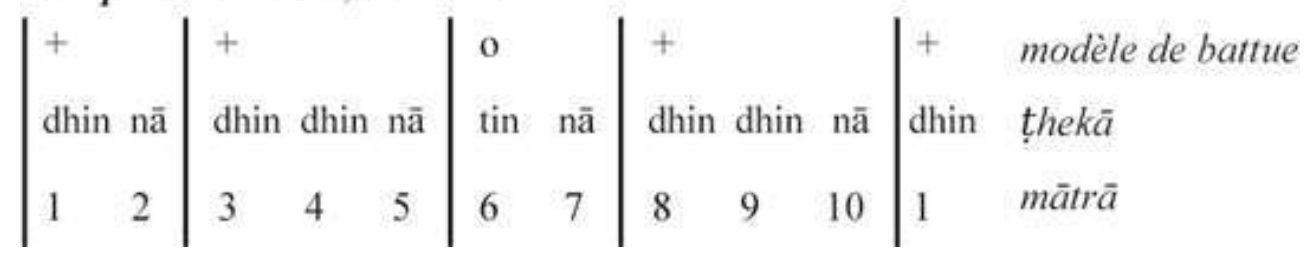

Ces deux systèmes de définition du tāl, le thekā et le modèle de battue coexistent, bien que l'un ou l'autre puisse avoir, selon le contexte, une plus grande importance pratique. Dans certains tâl, mais pas dans tous, il existe une corrélation évidente entre les implications structurelles du țhekā et de la battue. C'est le cas de jhaptāl où le geste marquant le « khāli » du troisième vibhāg (mātrā 6-7) correspond au «khālī» ou aux band bol (frappes dépourvues de résonances graves, représentées par des syllabes sourdes telles que tin et $n \bar{a})$. Cette corrélation n'est cependant pas toujours présente et ne correspond ni à une exigence théorique ni à une nécessité pratique. L'examen des raisons historiques de cet état de fait complexe sort du cadre de cet article.

D'autres termes définissant le rythme fournissent de nouvelles clés pour une compréhension de l'organisation rythmique dans la musique de l'Inde du Nord. Par exemple lay, le plus proche équivalent du terme occidental «tempo $»^{18}$, dont le champ sémantique couvre à la fois le tempo métrique (allure de la pulsation primaire), la densité rythmique $^{19}$ et la relation entre les deux ${ }^{20}$. Cette triple signification a évolué avec les changements intervenus dans la pratique au cours des deux derniers millénaires. En fait, dans son sens premier, lay désigne l'intervalle de temps entre deux pulsations là où ces pulsations étaient conçues comme des points dans le temps (potentiellement marqués par des actions) plutôt que des durées. Malgré d'énormes changements dans la théorie comme dans la pratique, certains aspects du rythme indien peuvent toujours être compris dans ce sens.

Un autre terme, chand, peut aussi se traduire par "mètre ", dans le sens du mètre prosodique (ce qui est son sens originel en hindi). Plus rarement utilisé que tāl, chand se réfère principalement aux schémas rythmiques caractéristiques d'un tāl (tel qu'il s'articule en particulier dans le țekā), surtout quand le schéma rythmique est reproduit hors de son contexte original. Par exemple, si le țhekā de jhaptāl est joué comme variation dans un solo de tablā d'un autre tāl (tel que tintāl à seize mātră), on peut parler d'un jeu en « jhaptāl chand »" 

métrique dans la musique de l'Inde du Nord. Le tāl a deux aspects, le premier est un schéma temporel abstrait qui se manifeste dans la battue (chironomie), le second est un motif rythmique répété qui est représenté par un thekā. Les schémas chironomiques extériorisent des structures temporelles abstraites, permettant à l'auditeur d'entendre la surface rythmique dans le contexte de ces cadres - un concept qui évoque fortement l'idée de Kolinski du mètre comme fournissant un «fond » sur lequel se déploient les " figures » du rythme. Cette interprétation est confirmée par l'utilisation du terme «lay », qui indique non seulement qu'il existe une ambiguïté inhérente au concept de «tempo » (comme en musique occidentale) mais aussi que la relation entre tāl et surface rythmique est susceptible d'être manipulée consciemment. L'histoire des spéculations théoriques sur le tāl montre que les musiciens indiens ont depuis longtemps perçu une distinction conceptuelle entre le tāl et le rythme.

Cependant l'utilisation de thekā et du terme « chand » laisse entendre que le tāl n'est pas un phénomène entièrement abstrait puisque les tāl sont associés à des motifs rythmiques concrets qui peuvent être reproduits même hors de leur contexte principal. Ceci suggère en réalité qu'un tāl est simplement un motif rythmique répété avec des paramètres d'accentuation, de durée et de timbre du tambour : quelque chose d'assez semblable à la «forme dynamique » décrite par Clynes et Walker ${ }^{22}$. Peut-être, comme le suggèrent ces auteurs, cette forme rythmique est-elle emmagasinée dans l'esprit de l'auditeur qui s'attend alors à ce que le motif soit répété et compare le nouveau rythme au motif qu'il attendait - d'où cette idée que la connaissance des divisions de la section théorique ( vibhāg) n'est peut-être pas indispensable.

Cette double nature du tāl en tant que structure temporelle et structure d'accentuation pourrait être mise en relation avec un autre domaine de recherche en psychologie musicale. Sur la base d'expériences faites avec des enfants, Bamberger démontre l'existence de deux modes de compréhension rythmique, qu'il appelle mode figuré et mode métrique. Ces modes sont décrits comme des exemples particuliers d'une dichotomie générale entre «modes figurés et modes formels du phénomène organisant le présent» (1991: 15). Selon cette théorie, la compréhension métrique dépend de la relation du rythme à une pulsation sous-jacente, alors que ce n'est pas le cas pour la représentation figurée, qui repose plutôt sur des principes généraux de la Gestalt tels que le groupement d'éléments semblables ${ }^{23}$.

28 Ceci nous rappelle que, même dans des cultures possédant des théories développées $d u$ mètre comme celle de l'Inde, il existe des façons non-métriques de comprendre le rythme. En d'autres termes, cela confirme l'idée que les tâl peuvent être identifiés sur la base de structures d'accentuation et/ou de variation de timbre, indépendamment du nombre d'unités temporelles - ce qui pourrait aider à expliquer les ressemblances entre le tīntāl à seize mātrā et ses différentes variantes, le dīpcandì à quatorze mātrā et le jhaptāl à dix mātrā : chacun ayant le même schéma chironomique noté $++0+$, et des séquences identiques de bol de tablā sans ses țhekăa ${ }^{24}$. Tout ceci témoigne de l'importance du schéma d'accentuation dans le tāl.

Le tâl peut donc être conçu comme une notion soit abstraite soit concrète. Et souvent les deux à la fois, d'où la correspondance qui survient parfois entre la structure chironomique et le țhekāa - auquel cas, le țhekā rajoute un élément audible au schéma temporel abstrait. Très souvent cependant, c'est l'un des deux qui prédomine, la structure chironomique ou le țhekā, l'autre étant presque totalement dénué de pertinence.

Cahiers d'ethnomusicologie, 10 | 1997 


\section{Comparaison entre le tāl et le mètre : remarques préliminaires}

Comment établir un lien entre le tāl et les théories du mètre décrites plus haut? Nous avons déjà constaté que le tāl semble confirmer l'idée de Kolinski inspirée de la Gestalt psychologie, qu'on le comprenne comme cadre abstrait ou même comme une structure rythmique concrète. En ce sens, le tâl serait une forme spéciale du mètre, un cadre temporel fonctionnant comme arrière-plan au dessin rythmique. La théorie métrique de Lerdahl et Jackendoff semble aussi s'appliquer au tāl, qui s'analyse parfaitement dans ces termes. La seule chose à laquelle il faut être attentif est que le niveau médian de pulsation (le vibhāg ou section) peut être irrégulier, comme c'est le cas pour jhaptāl. Si l'on considère le mātrā comme étant le plus haut des niveaux de pulsation métriquement signifiants, le vibhāg comme le second et l'āvārt comme le troisième et le plus faible, on peut appliquer la notation de Lerdahl et Jackendoff comme suit.

Les pulsations sont représentées par des points sur trois niveaux : les points ressentis comme des pulsations sur les trois niveaux sont structurellement les plus forts (sam); viennent ensuite ceux qui sont perçus sur deux niveaux (mātrā 3, 6 et 8); les plus faibles sont ceux qui n'interviennent que sur un seul niveau (mātrā 2, 4, 5, 7, 9 et 10).

Fig. 4 : Analyse de jhaptāl selon la théorie métrique de Lerdahl et Jackendoff

Jhaptäl: 10 mātrā, 2+3+2+3

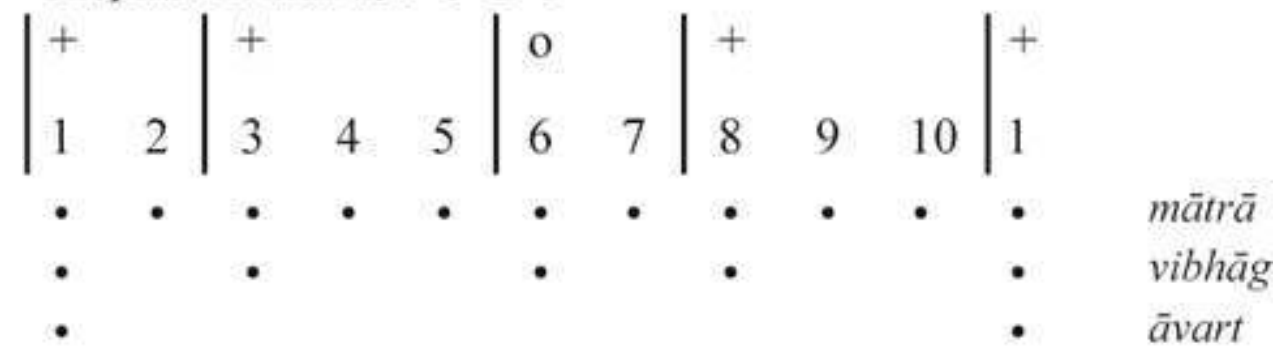

Chaque tāl présente donc trois niveaux de pulsation distincts et interdépendants, le mātrā , le vibhāg et l'āvārt. (En fait, dans le cas de tāl symétriques comme jhaptāl où la deuxième partie du cycle se distingue de la première par la présence du geste muet khālīi il y aurait de bonnes raisons d'inclure un quatrième niveau, le demi cycle. Nous ne l'avons pas fait ici pour ne pas compliquer la démonstration.) En combinant la théorie de Lerdahl et Jackendoff à celle de l'Inde, on peut dire que les trois niveaux de pulsation qui définissent le tāl se combinent pour former un cadre métrique consistant.

Si l'on compare ce qui a été dit du tāl et des quatre hypothèses de travail concernant le mètre, on peut faire les remarques suivantes:

1. Le tāl, comme le mètre, est un cadre temporel périodique et hiérarchique. On peut supposer qu'une représentation cognitive de ce cadre est construite par l'auditeur (dans la mesure de ses connaissances et de son expérience).

2. Comme le mètre, le tâl implique l'interaction de deux courants de pulsation ou plus (en fait, d'habitude au moins trois). Un de ces niveaux de pulsation peut toutefois être composé d'une séquence d'intervalles de temps d'inégales longueurs. Les pulsations sur plus d'un seul niveau sont importantes structurellement et marquées par des gestes de la main. 
3. Comme le mètre, le rythme dans la musique indienne est interprété en fonction du tâl. Cependant ce que révèle le tāl pourrait être moins subjectif que certaines autres formes métriques, surtout quand la structure du tāl est clairement illustrée par la chironomie.

4. Ce que les auditeurs sans connaissances musicales peuvent déduire à partir du tāl doit être, en admettant que ce soit possible, au moins aussi complexe que pour le mètre. Quant aux auditeurs dotés d'une culture musicale, la démarche est relativement simple, elle consiste essentiellement à reconnaître le motif chironomique et /ou le thekā.

En général il semble que le tāl de l'Inde du Nord fonctionne à beaucoup d'égard comme le mètre occidental, et ceci de deux façons principales - il peut avoir un niveau de pulsation intermédiaire irrégulier et il est d'habitude déterminé de manière absolument objective par la convention. Ce dernier fait ne constitue peut-être pas une distinction nette par rapport à la musique occidentale ; il s'agit plutôt d'une différence de degré : jhaptāl a dix $m \bar{a} t r a \bar{a}$ (toujours dix et non un multiple ou un facteur de dix), il ne peut y avoir d'ambiguïté sur l'endroit où intervient le premier temps (sam) etc. Des indications audibles et visibles permettent à l'auditeur de ne pas se tromper sur le tâl et au musicien de ne pas perdre, par inadvertance, le compte des battements (ce qui arrive parfois, bien que dans la pratique de telles erreurs soient extrêmement rares, même pour les tāl les plus complexes).

Comparé à beaucoup d'autres systèmes métriques, le tāl est particulièrement explicite. Il n'est en effet pas une simple qualité inhérente à la musique que l'auditeur déduit sans le secours d'aucune aide. Si tel était le cas, les auditeurs pourraient trouver plusieurs interprétations métriques valables pour une seule pièce, une idée totalement étrangère à la pensée musicale indienne. Dans la musique indienne, il n'est pas rare qu'on engage un musicien, principalement ou même exclusivement, pour garder le tāl (comme c'est le cas pour le joueur de tablā lui-même dans certains genres de la musique de l'Inde du Nord). Les chanteurs comptent le tāl, une partie des auditeurs se joignent à eux et il n'y a pas de choix ou de subjectivité qui intervienne dans l'interprétation métrique. La raison profonde de ce phénomène se trouve, disons-le en passant, dans la nature rituelle ou quasi-rituelle de la musique indienne à ses origines, un sujet que nous ne pouvons examiner ici en détail.

\section{L'importance du tempo (lay) pour déterminer la structure métrique}

Revenons à notre modèle d'un cadre de tāl comportant trois ou plus de trois niveaux de pulsations et fonctionnant comme matrice de référence pour le rythme de surface. On peut illustrer ce modèle par une série de points comme c'est le cas ci-dessous. Dans la pratique indienne, le rythme de surface n'est pas seulement interprété en fonction du tāl, il est souvent produit directement par une subdivision de la battue des mātrā. C'est pourquoi, dans le schéma suivant, ce niveau de surface est indiqué par une autre rangée de points. 
Fig. 5 : Un modèle de tāl comme interaction de trois niveaux de pulsation et sa relation avec le rythme de surface (toujours pour le jhaptā).

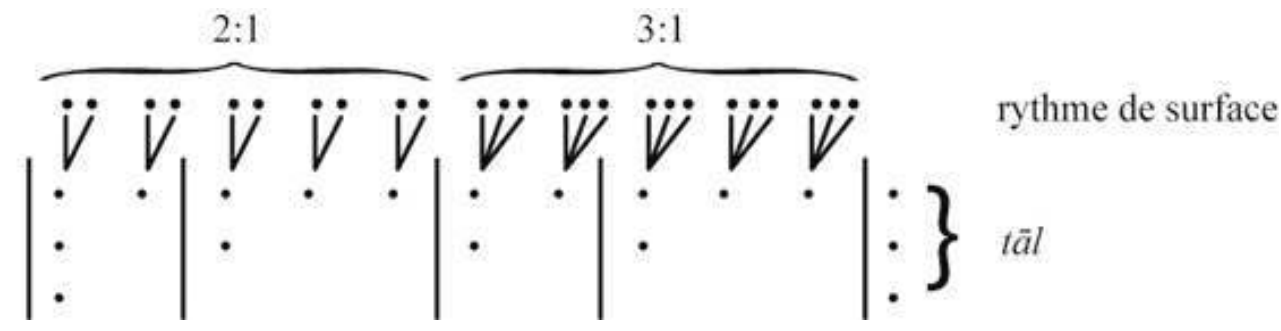

Ce modèle est valable pour une bonne partie de la musique de l'Inde du Nord, tant vocale qu'instrumentale et, quand c'est le cas, il se révèle être un instrument d'analyse fort utile. Toutefois, dans nos recherches, nous sommes tombés sur plusieurs exemples pour lesquels ce modèle ne fonctionne apparemment pas. Ou alors il fonctionne, mais au prix de modifications considérables. L'explication de ce phénomène semble être dans le tempo adapté.

Le modèle ci-dessus suggère des fonctions nettement définies pour les différents niveaux de pulsation. Le mātrā est la pulsation métriquement signifiante la plus rapide - l'allure de pulsation primaire qui définit le tempo. Les vibhāg regroupent les mātrā et assignent leurs fonctions aux groupes, transformant un simple courant de pulsations en un mètre où certains mātrā sont accentués. La séquence des vibhāg est ce qui est marqué par la battue chironomique. L'āvārt est la pulsation métrique signifiante la plus lente; cependant son sens est généralement sensiblement différent en ceci qu'il est la plus longue unitétemporelle signifiante dans l'organisation rythmique.

Le fonctionnement de chaque niveau implique des limites à la vitesse ou à l'allure de chaque pulsation. En particulier, si l'on accepte ce modèle, le mātrā devrait être assez rapide pour être perçu comme un courant de pulsation relié plutôt que comme des événements indépendants, mais assez lents pour pouvoir être comptés confortablement. Nous ne voulons pas donner ici de limites métronomiques strictes; mentionnons cependant, comme référence, la suggestion de Sachs souvent invoquée bien que d'une précision peut-être excessive qui donne pour limites naturelles du tempo 32-132 MM (1953 : 33). Handel, sur une base plus scientifique suggère qu'avec des intervalles « de plus 1.5 secondes, deux éléments perdent leur sens de cohérence et semblent sans liens l'un avec l'autre » (1989: 389), ce qui implique un chiffre minimum d'à peu près $40 \mathrm{MM}$ pour le tempo. Le vibhāg devrait aussi entrer dans ces limites. D'autre part, la plus longue unité de temps signifiante du point de vue métrique devrait tenir compte des limites de notre perception du présent. Si cette hypothèse est correcte, le cycle (āvārt) ne devrait pas dépasser une durée d'une quinzaine de secondes ${ }^{25}$.

On peut prédire que, si le tempo s'écarte trop de ces limites notionnelles, leur fonction et peut-être même la texture tout entière du système du tāl s'en trouveront altérées. Or c'est exactement ce qui se passe dans la pratique, et les conséquences sont tout à fait celles qu'on pouvait prévoir.

41 Les changements intervenus dans la pratique de la musique hindoustanie ces dernières cent cinquante à deux cents dernières années se sont traduites par l'utilisation d'un très grand éventail de tempos de mātrā: mesurée en mātrā par minutes, la fourchette s'étend d'à peu près $10 \mathrm{MM}$ à bien au delà de $700 \mathrm{MM}^{26}$. On est en droit de se demander s'il ne s'agit pas là d'un éventail trop étendu pour les tempos, ce qui entraîne des longueurs de 
cycles allant d'à peu près $1.5 \mathrm{~s}$. à plus d'une minute. L'éventail des tempos est donc trop large pour que le tāl puisse fonctionner en pratique comme dans le schéma donné cidessus - quand il est très rapide, l'allure des mātrā est trop grande pour fonctionner métriquement; quand il est très lent, le cycle est trop long pour être retenu par le présent perceptuel (voir fig. 6).

Fig. 6 : À gauche, comparaison des longueurs typiques d'un cycle (āvārt) avec l'étendue du présent perceptuel ; à droite, allures typiques de mātrā avec une estimation de l'allure des pulsations définissant le tempo.

\section{Unité de temps (s)}

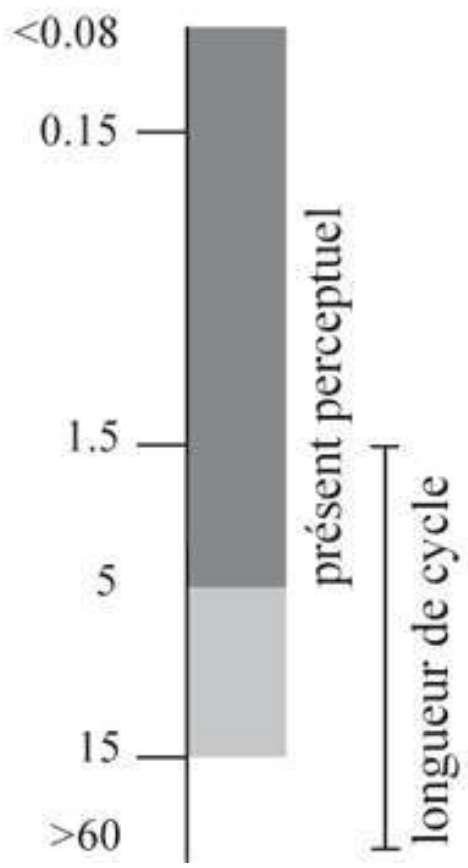

\section{allure de pulsation (MM)}

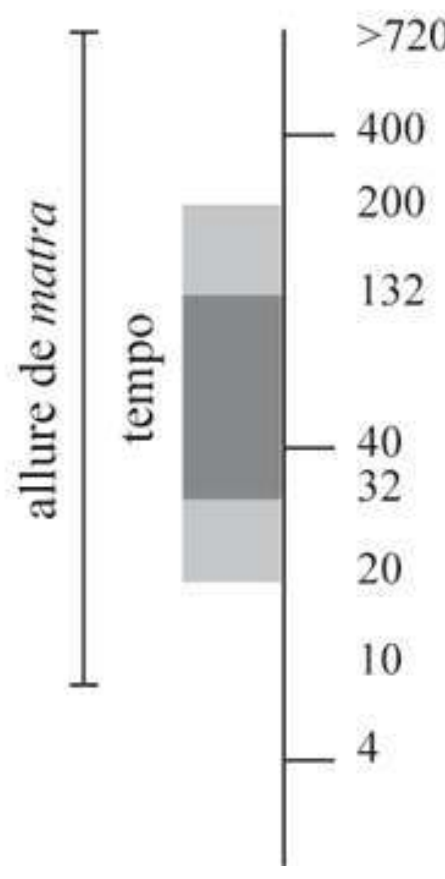

Dans la pratique, ce qui se passe quand les tempos sont très lents est que le mātrā devient trop long pour être perçu comme pulsation unité de temps - les musiciens comptent alors par unités d'un demi ou d'un quart de mātrā et les joueurs de tablā articulent ces divisions quand ils jouent le thekā. En pratique, la pulsation d'un demi ou d'un quart de mātrā joue le rôle généralement dévolu au mātrā et le mātrā y joue celui du vibhāg (dans les tempos très lents la chironomie intervient rarement, ce qui rend plus aisée l'usurpation de la fonction de vibhāg). Le vibhāg ne peut cependant être considéré comme remplissant la fonction de l'āvārt étant donné l'importance que conserve la première frappe (sam), aussi bien dans les compositions que dans l'improvisation, sans compter que le thekā ne se répète pas au niveau du vibhāg. Le cycle conserve un sens en tant qu'unité, mais se trouve dissocié de la structure métrique fonctionnelle parce que la durée est bien plus grande que le présent perceptuel. La structure du mètre se trouve donc distordue par le tempo lent comme le montre le schéma suivant (fig. 7). 
Fig. 7 : L'expansion de tintā 27 à seize mātrā en un tempo de vingt-quatre mātrās par minute. $\mathrm{m}=$ mātrā, v = vibhāg, a = āvārt ; l'allure des pulsations est donnée en MM. Le niveau le plus bas, l'āvārt, est dissocié de la structure métrique au tempo lent (vilambit lay).

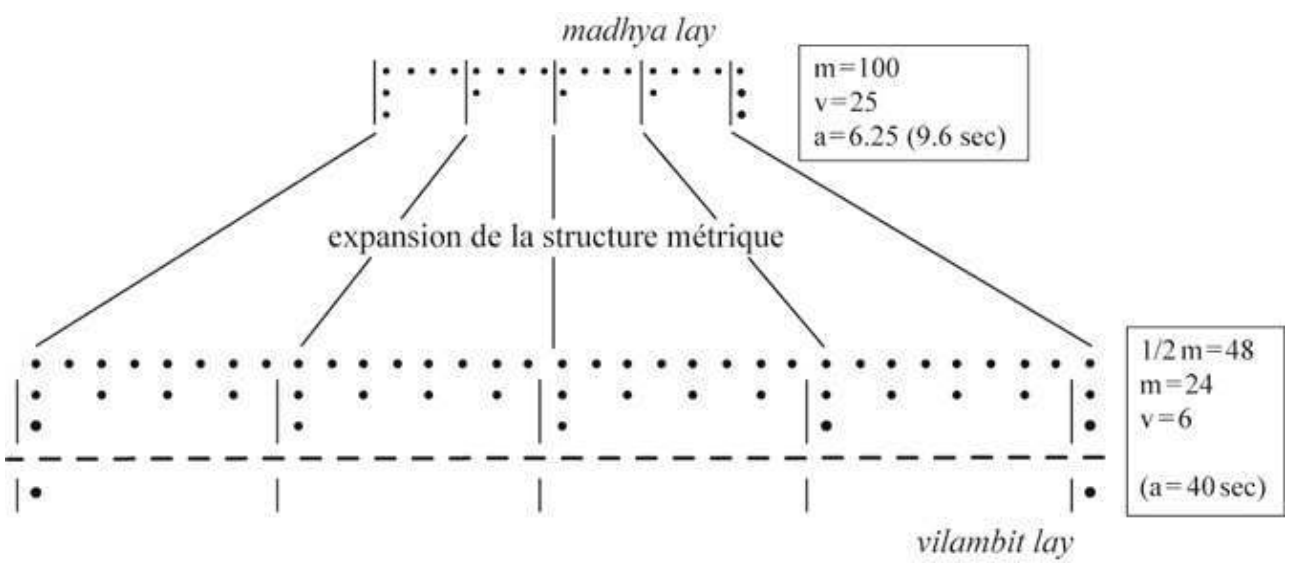

L'inverse se passe lorsque le tempo est très rapide : le mātrā devient alors identique au niveau du rythme de surface, puisqu'il s'approche de la vitesse maximale de l'articulation du soliste et ne peut donc plus être subdivisé ; mais il est trop rapide (700 MM+ parfois) pour être considéré comme l'allure de pulsation primaire. C'est le vibhāg qui joue alors ce rôle en conservant cependant sa fonction dans la chironomie ; l'āvārt, quant à lui, reste la durée métriquement signifiante la plus longue (des durées plus importantes que l'āvārt ont rarement une signification métrique dans la musique savante de l'Inde du Nord). Le tâl s'est donc simplifié en passant d'une structure à trois niveaux à une structure à deux niveaux seulement ${ }^{28}$.

Fig. 8 : La compression du tintāl à des tempos très élevés. Le niveau le plus élevé, le mātrā, perd sa fonction à un tempo très élevé (ati-drut lay).

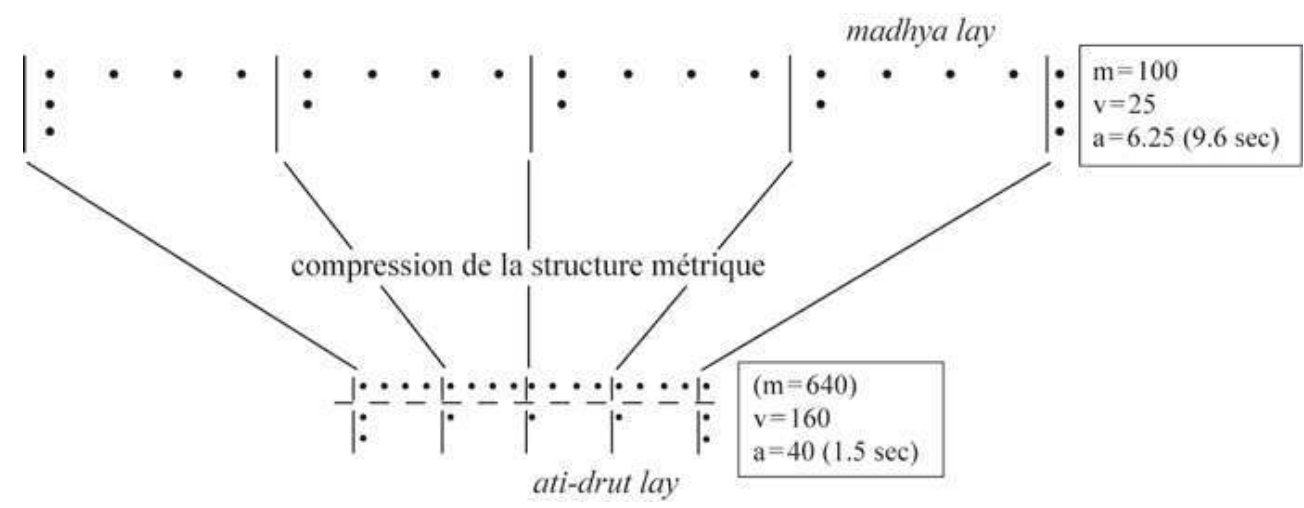

Ce déplacement dans la fonction métrique a des conséquences sur la façon de mesurer le tempo : dans les tâl très lents, l'unité la plus appropriée devient un demi ou un quart de $m \bar{a} t r a \bar{a}$; dans les tāl très rapides, elle comporte deux, trois voire quatre pulsations de mātrā . Même mesuré de cette façon, l'éventail des tempos en usage dans la musique de l'Inde du Nord reste très grand ${ }^{29}$, d'une étendue qui atteint peut-être de 20 à $180 \mathrm{MM}$, ce qui est possible à cause de la nature très développée du système du tāl et de sa théorie permettant la représentation de structures qui, autrement, seraient difficiles à percevoir à cause des tempos extrêmes. 


\section{Le mètre dans la musique sans tāl} Nous avons commencé avec un double modèle du mètre, comme interaction de plusieurs niveaux de pulsation tout d'abord (en remplacement d'une idée plus ancienne mais plus problématique du mètre comme alternance entre temps forts et temps faibles) et comme arrière-plan au dessin rythmique. Ces deux aspects s'appliquent sans aucun doute à la musique de l'Inde du Nord, non seulement pour toutes les formes rattachées au tāl, mais 
encore pour beaucoup de musiques sans tāl. Le tāl lui-même a cependant des exigences plus contraignantes.

1. Le tāl est une dimension explicite de la musique qui doit être établie sans ambiguïté.

2. Le rythme doit être organisé selon des structures métriques autorisées ou tâl.

3. Ces tăl sont des structures temporelles où se recoupent au moins trois (et non deux) niveaux de pulsation.

Dans le cycle du tāl, la définition de temps forts ou faibles serait problématique: on pourrait croire en effet que les temps forts sont accentués du point de vue dynamique, c'est-à-dire plus sonores que le reste. En fait, dans la musique indienne, les accents dynamiques peuvent intervenir sur n'importe quel temps (ou même entre ceux-ci). Ce que donne le tāl est une matrice temporelle structurellement importante, qui intervient quand les niveaux de pulsations coïncident sur deux niveaux ou plus : ces pulsations n'ont pas besoin d'être accentuées mais peuvent être déduites, par exemple, des changements de timbre au tambour ou de structure de composition.

La musique de l'Inde du Nord vient aussi clairement étayer les théories de Kolinski dans la mesure où les musiciens indiens font une distinction conceptuelle nette entre le rythme et le mètre, ce qui évoque le rapport de la forme au fond dans la Gestalt Psychologie. Le mètre (tâl) est un cadre abstrait, tout d'abord construit dans l'esprit du musicien et représenté dans la chironomie et/ou le țhekā, d'où il peut être reconstruit par l'auditeur. Le rythme est compris dans le contexte de ce cadre et, bien qu'il puisse souvent être directement généré par le tāl, il reste séparé de celui-ci.

51 Cette séparation pose cependant certains problèmes. Par exemple, le thekā est une structure rythmique utilisée pour représenter un mètre. Comme il a cette fonction, il finit par être identifié au mètre. Ainsi la distinction entre le rythme et le tâl et la nature abstraite de ce dernier peuvent être mises en danger par l'utilisation du thekā. Il est fréquent dans de nombreuses traditions musicales que le mètre soit défini par une structure rythmique qui se répète, et pour ceux qui pratiquent ces musiques, l'idée d'un mètre sans rythme n'aurait probablement pas beaucoup de sens. (Cette proximité crée une confusion sur le plan théorique, à tel point que dans certains travaux musicologiques, la distinction entre rythme et mètre est des plus vagues, voire inexistante).

Cette étude rappelle aussi l'importance cruciale du tempo dans le fonctionnement du mètre. Le mètre est formé par l'interaction de niveaux de pulsations et il est probable que ces derniers ont certaines fonctions dans la représentation cognitive du mètre. Les mètres peuvent être accélérés ou ralentis; mais si le tempo était modifié au delà de certaines limites (et l'on ne peut que deviner pour l'instant ce qu'elles pourraient être), le fonctionnement du mètre s'en trouverait altéré. La fonction de certains niveaux de pulsation peut changer et devenir obsolète du point de vue métrique, ou alors de nouveaux niveaux peuvent entrer en jeu.

53 Un autre domaine dans lequel la musique indienne peut nous aider dans la réflexion sur le mètre est l'importance de la théorie : le système métrique de l'Inde du Nord ne pourrait certainement pas exister dans sa forme actuelle sans la contribution de la théorie au développement de la pratique ; de même, bon nombre de structures métriques complexes ne pourraient être perçues par des auditeurs s'ils n'avaient aucun accès à ces mêmes théories. La théorie n'est donc pas seulement quelque chose qui décrit le tāl, ne serait-ce qu'imparfaitement; elle fait partie de la texture même du tāl et ne peut être dissociée de 
sa pratique. La théorie aide à la représentation cognitive des structures complexes et crée des possibilités de développements dans la pratique.

Cette étude montre qu'il existe de nombreuses ressemblances entre le concept de mètre tel que le comprennent les musicologues occidentaux et celui de tāl comme il s'est développé en Inde. Les différences - l'utilisation de niveaux de pulsation irréguliers en Inde, le fait qu'on insiste sur trois niveaux de pulsation ou plus, ainsi que la nature explicite du tāl - sont réelles. Mais, compte tenu des ressemblances fondamentales qui existent entre les deux concepts, ces différences sont relativement superficielles. Il s'agit là d'une observation importante car elle implique que, si le mètre n'est pas présent dans toutes les musiques, il n'est pas non plus limité à la musique occidentale. Nous pensons qu'une réflexion sur la musique indienne permet à notre concept de mètre d'être appréhendé dans une perspective différente et utile, et vice versa.

\section{BIBLIOGRAPHIE}

AGAWU Kofi, 1995, Africa rhythm. A Northern Ewe perspective. Cambridge : Cambridge University Press

AROM Simha, 1991, African polyphony and polyrythm. (trans. M. Thom, B. Tuckett, R. Boyd)

Cambridge : Cambridge University Press.

BAMBERGER Jeanne, 1991, The mind behind the musical ear. How children develop musical intelligence. Cambridge, Mass. : Harvard University Press.

BERRY Wallace, 1976, Structural functions in music. Englewood Cliffs : Prentice-Hall.

CLAYTON Martin R. L., 1993a, The rhythmic organisation of North Indian classical music : tāl, lay and laykārî. Unpub. PhD diss., SOAS, University of London.

CLAYTON Martin R. L., 1993b, « Two gat forms for the sitār : a case study in the rhythmic analysis of North Indian music ». British Journal of Ethnomusicology 2, pp. 75-98.

CLAYTON Martin R. L., 1996, « Free rhythm : ethnomusicology and the study of music without metre » Bulletin of SOAS. LIX/2, pp. 323-332.

CLYNES Manfred et WALKER Janice, 1982, « Neurobiologic functions of rhythm, time and pulse in music » in Clynes, M (ed.) Music, Mind and Brain. pp. 171-216. New York : Plenum Press.

COOPER Grosvenor et MEYER Leonard B., 1960, The rhythmic structure of music. Chicago : University of Chicago Press.

DOWLING W. Jay and HARWOOD Dane L., 1986, Music cognition. London : Academic Press.

GOTTLIEB Robert, 1993, Solo tablā drumming of North India : its repertories, styles and performance practices. (2 vols). Delhi : Motilal Banarsidass.

GROVE 6, 1980, The New Grove dictionary of music and musicians. ed. Stanley Sadie, London : Macmillan 
HANDEL Stephen, 1989, Listening : an introduction to the perception of auditory events. Cambridge, Mass. : MIT Press.

HOPKINS Pandora, 1982, « Aural thinking » in R. Falck and T. Rice (eds) Cross cultural perspectives on music. Toronto : University of Toronto Press.

KIPPEN James R., 1988, The tablā of Lucknow : a cultural analysis of a musical tradition. Cambridge : Cambridge University Press.

KOLINSKI Mieczyslaw, 1959, « The evaluation of tempo » Ethnomusicology 3/2 : 45-47.

KOLINSKI Mieczyslaw, 1973, «A cross-cultural approach to metro-rhythmic patterns » Ethnomusicology $17 / 3$ : 494-506.

LERDAHL Fred et JACKENDOFF Ray, 1983, A generative theory of tonal music. Cambridge, Mass. : MIT Press.

MANUEL Peter, 1983, « The concept of Tala in semi-classical music ». National Centre for the Performing Arts Quarterly Journal (Bombay) 12/4 : 7-14.

PANTALEONI Hewitt, 1987, « One of Densmore's Dakota rhythms reconsidered » Ethnomusicology $31 / 1: 35-55$.

ROWELL Lewis, 1988, « Form in the ritual theatre music of ancient India » in Musica Asiatica 5 Cambridge : Cambridge University Press.

SACHS Curt, 1953, Rhythm and Tempo. London : Dent.

SMITH K. C., CUDDY L. L. et UPITIS R., 1994, « Figural and metric understanding of rhythm ». Psychology of Music 22 : 117-135.

VOS P. G., 1979, Identification of metre in music. (Internal Report 0N07). Nijmegen : University of Nijmegen.

WALKER Robert, 1992, Review of Bamberger 1991. Psychology of Music 20 : 186-191

WIDDESS Richard, 1994, «Involving the performers in transcription and analysis : a collaborative approach to dhrupad » Ethnomusicology 38/1:59-80

WINDSOR W. Luke, 1993, « Dynamic accents and the categorical perception of metre » Psychology of Music $21: 127-140$.

YESTON Mamy, 1976, The stratification of musical rhythm. New Haven, Conn. : Yale University Press.

\section{NOTES}

2. Clayton 1993a (partiellement résumé dans 1993b). Une version révisée de ce travail doit être publiée par Oxford University Press.

3. Nous remercions Richard Widdess et Jan Gross pour leurs commentaires sur des versions antérieures de cet article.

4. En fait la notion de "pulsation » est un peu ambiguë puisqu'elle se réfère à la fois à une unité de temps et à un point dans le temps (et même à une action qui a lieu à ce point).

5. Voir par exemple Cooper et Meyer (1960:4), qui adoptent la première hypothèse, Grove 6 $(1980$, vol $12: 222)$ qui suit la seconde ; et les définitions plus sophistiquées de Yeston (1976:32-3) et Lerdahl et Jackendoff (1983: 12).

6. Comme ont commencé à le faire plusieurs musicologues : voir Lerdahl et Jackendoff (1983), Yeston (1976) etc. 
7. Par exemple, Berry suggère qu' «il est fondamental que le mètre soit souvent indépendant de la barre de mesure ", et qu'un aspect important de l'analyse métrique est de déterminer le mètre réel, qu'il corresponde ou non avec le mètre noté (1976 : 324).

8. Les ethnomusicologues qui ont écrit sur ces problèmes sont Kolinski (1973), Pantaleoni (1987) et Arom (1991).

9. Pour une réflexion et une bibliographie sélective sur le rythme libre, voir Clayton 1996.

10. Voir par exemple Agawu $1995: 185$ et suivantes.

11. Voir aussi Dowling et Harwood $1986: 186$.

12. Voir Clayton 1993a : 44 et suivantes.

13. Voir par exemple Yeston $1976: 32-3$.

14. La subjectivité de l'interprétation métrique dans la pratique est démontrée de différentes manières par Hopkins (1982) et par Vos (1979, cité par Handel 1989 : 411 et suivantes); le sujet mériterait cependant une étude plus approfondie.

15. Le mot «rythme » est utilisé ici dans son sens général d'arrangement d'éléments musicaux dans le temps.

16. Voir par exemple Windsor (1993) pour une évaluation de l'interdépendance des accents dynamiques et de la structure rythmique.

17. Le sam a cependant un signe de notation, « $\mathrm{X} »$. Les conventions d'écriture varient : les tālî, par exemple, peuvent être indiqués par des chiffres plutôt que simplement par «+».

18. Le terme lay est aussi utilisé par les auteurs indiens comme l'équivalent le plus proche du mot "rythme».

19. L'allure à laquelle arrivent les événements rythmiques : le « tempo » dans le sens où l'entend Kolinski dans son article de 1957.

20. Rowell écrit que dans l'ancienne théorie indienne le lay ne représentait " ni 'l'allure de pulsation', ni 'l'allure à laquelle arrivent les événements' comme on le conçoit en Occident ; il serait plus juste de le voir comme le taux de succession structurale tel qu'il est mesuré et réglé par les structures gestuelles sous-jacentes... » (1988:150). Utilisé par les musiciens hindoustani modernes, le lay peut se référer à chacun de ces concepts.

21. Voir Kippen (1988: 169-170) et Gottlieb (1993 : vol 1, p. 133, vol 2. p. 140).

22. Clynes et Walker parlent d'une « reproduction d'une seule forme dynamique engrangée dans la mémoire» (1982: 176). Ce concept semble convenir aux tāl, lorsqu'ils sont soutendus en pratique par un motif répété au tambour avec peu ou pas de variations.

23. Certains éléments de la théorie de Bamberger sont contestés - dans ce cas précis ses travaux ont été critiqués pour leur manque de perspective culturelle (voir Walker 1992). Le sens qu'elle donne aux modes figurés et métriques est cependant ce qui nous intéresse ici - notre idée étant qu'il existe peut-être une corrélation entre la double nature de la compréhension rythmique telle qu'elle est formulée par Bamberger - et développée par d'autres tels que Smith, Cuddy et Upitis 1994 - et celle du tāl tel qu'il est décrit ici.

24. Manuel décrit avec plus de détails les relations entre ces tāl en remarquant que des tâl tels que dīpcandī, cañcar et $a d d h \bar{a}$ peuvent correspondre à des structures soit à quatorze, soit à seize temps (1983).

25. La psychologie cognitive ne donne pas de maximum absolu pour ce présent perçu ou présent psychologique (qui, pense-t-on, correspond de près aux limites de la mémoire de travail). Dans des conditions normales, il s'étend de deux à cinq secondes, bien qu'on admette que, dans certains cas, il puisse être augmenté. Cependant même alors, il est peu probable qu'il s'étende au delà d'à peu près douze à quinze secondes.

26. Des tempos très lents sont entrés dans les mœurs surtout dans la forme vocale du barā khyāl, alors que des tempos très rapides sont apparus surtout dans la musique instrumentale; l'essentiel de ces changements est intervenu au cours de ce siècle. Le fait que ces développements 
soient assez récents confirme l'idée que le modèle de tāl décrit plus haut est prédominant du point de vue historique avec des développements tardifs, venant altérer sa fonction.

27. Un cycle de tīntāl comporte seize mātrā, divisés en quatre vibhāg de quatre mātrā chacun.

28. Ou de quatre à trois niveaux, si le niveau du demi-cycle est considéré comme signifiant du point de vue métrique.

29. Comparé à la suggestion de Sachs : voir la note ci-dessus.

30. Deepak Choudhury (communication personnelle). Certains chanteurs de dhrupad utilisent aussi cette pratique, tandis que les joueurs de rudra vînā demandent à leur percussionniste au pakhavaj (tambour tubulaire) de jouer le cautāl à douze mātrāa pendant la section jor (communication personnelle de R. Widdess).

\section{RÉSUMÉS}

Cet article traite de la relation entre le concept de tāl dans la musique hindoustānī de l'Inde du Nord et celui de mètre dans la musique occidentale et en ethnomusicologie en établissant des rapprochements entre différentes branches de la recherche musicale. Qu'entend-on par mètre, qu'est-ce que le tāl, et dans quelle mesure ces deux concepts se recoupent-ils ? La musicologie, l'ethnomusicologie et la psychologie musicale fournissent-elles des clés pour comprendre la musique indienne, et l'étude du tāl permet-elle d'envisager la notion de mètre comme un phénomène musical largement répandu? En plus d'une réflexion sur ces questions théoriques, cet article propose quelques exemples concrets des bénéfices à tirer d'une approche interdisciplinaire.

This paper discusses the relationship between the concept of tāl in North Indian Hindustani music, to that of metre in Western music and in ethnomusicology, drawing together strands of research from several different branches of music studies. What do we mean by metre, what is tāl , and to what extent do the two concepts overlap? Can studies in musicology, ethnomusicology and music psychology provide insights into Indian music, and can the study of tâl contribute to an understanding of metre as a widespread musical phenomenon? Besides discussing some of the theoretical issues generated by such questions, this paper also provides some concrete illustrations of the benefits of interdisciplinarity and methodical comparison.

\section{AUTEURS}

\section{MARTIN CLAYTON}

Martin CLAYTON a entrepris l'étude de la musique savante de l'Inde du Nord en 1984 lors d'un séjour à Delhi. En 1988 il obtient son BA (en musique et en hindi) à la School of Oriental and African Studies de l'Université de Londres; de 1989 à 1992, il prépare son PhD dans la même institution. Il a ensuite enseigné l'ethnomusicologie, la musique indienne et donné des cours de sitar dans différentes universités du Royaume-Uni. Membre du comité du British Forum for Ethnomusicology, Martin Clayton est à l'heure actuelle maître de conférence en ethnomusicologie à l'Open University. 